\title{
Chirurgia bariatrica versus terapia medica intensiva per il diabete: outcome a 3 anni
}

\author{
Paola Fierabracci
}

Pubblicato online: 10 luglio 2014

(C) Springer International Publishing AG 2014

\section{Commento a:}

Bariatric surgery versus intensive medical therapy for diabetes-3-year outcomes.

P.R. Schauer, D.L. Bhatt, J.P. Kirwan, K. Wolski, S.A. Brethauer, S.D. Navaneethan, A. Aminian, C.E. Pothier, E.S. Kim, S.E. Nissen, S.R. Kashyap, for the

STAMPEDE Investigators.

N Engl J Med (2014) 370:2002-2013

In questo studio sono stati randomizzati 150 soggetti obesi di età media di 48 anni (68\% donne), con diabete tipo II non compensato per essere sottoposti a terapia medica intensiva da sola o associata a bypass gastrico (BG) o gastrectomia a manica (SG). L'end-point primario era di ottenere un'emoglobina glicata inferiore al 6\% a 3 anni dalla randomizzazione. L'indice di massa corporeo medio era 36 e il valore medio dell'emoglobina glicata alla randomizzazione era di $9,3 \%$. Circa il $91 \%$ dei soggetti completava lo studio a 36 mesi. Dopo 3 anni, il criterio per l'end-point primario nel caso della sola terapia medica veniva raggiunto nel 5\% dei pazienti, nei soggetti sottoposti a gastrectomia a manica saliva al $24 \%$ e in quelli trattati con bypass gastrico raggiungeva il 38\%. Nei soggetti sottoposti a chirurgia bariatrica l'uso di farmaci antidiabetici compresa l'insulina era inferiore; il calo ponderale ottenuto era del $25 \%$ nel gruppo sottoposto a bypass gastrico, del $21 \%$ nei soggetti trattati con gastrectomia a manica e del $4 \%$ nei soggetti trattati con tera- pia medica. Nei soggetti chirurgici la qualità della vita risultava migliore. Gli autori concludono che, nei soggetti obesi diabetici con scompenso glicometabolico, la terapia medica intensiva è molto più efficace se associata alla chirurgia bariatrica nel controllo glicemico, anche nel lungo tempo.

Nel trial STAMPEDE (Surgical Treatment and Medications Potentially Eradicate Diabetes Efficiently) gli autori avevano concluso che a 1 anno dalla randomizzazione il BG e la SG erano superiori rispetto alla terapia medica nell'ottenere un controllo glicemico adeguato e nel ridurre il rischio cardiovascolare e in questo studio gli stessi autori si proponevano di verificare se i benefici metabolici della chirurgia bariatrica erano duraturi nel più lungo termine e si accompagnavano a un miglioramento della qualità della vita. A differenza di altri studi precedenti che confrontavano la terapia antidiabetica convenzionale con la chirurgia bariatrica e che comunque riportavano effetti migliori di quest'ultima sul controllo glicemico, in questo studio sono stati selezionati soggetti con diabete scompensato e di lunga durata, per cui i risultati ottenuti indicano che anche in questo gruppo di soggetti i benefici della terapia bariatrica nel medio termine sono superiori rispetto alla terapia medica intensiva.

Questi risultati che confermano l'efficacia della chirurgia bariatrica nell'indurre la remissione e/o il miglioramento del diabete tipo II associato all'obesità dovranno essere valutati nel più lungo termine anche riguardo l'incidenza della patologia micro-e macrovascolare.

P. Fierabracci $(\square)$

UO Endocrinologia I, Azienda Ospedaliero Universitaria Pisana,

Pisa, Italia

e-mail: pfierab2001@yahoo.it 